\title{
dique seco de carena
}

\author{
Información facilitada por Northwest Division - Bureau of Yards and Docks
}

simopsis

$553-3$

Los grandes portaviones modernos tienen tales dimensiones que dificultan su reparación en los diques normales. Esto ha exigido la construcción de un nuevo dique capaz para aquel tipo de barcos, cuyas dimensiones son $360 \mathrm{~m}$ de $10 n g i-$ En la primera fase de estos trabajos se construyó un muelle de trabajo, de unos $48 \mathrm{~m}$ de Armada dispone en Bremerton. dique. En una segunda fase se construirá un muelle de atraque. Las modernas instalaciones con cada lado del futuro dique cubren todas las necesidades principales que este tipo de reparaciones requiere, lo que con que cuenta el nuevo plejo operacional económico y eficiente. Se estudiaron varias soluciones y lugares de emplazamiento, ya que el fondo de la bahía presenta varias capas de fangos y arenas de distinta granulometría que hacían prever posibles dificultades para cimentar. La capa superior admitida como base aceptable para la sustentación de la obra, está constituida por un banco sedimentario de detritus glaciares. El lugar elegido para la ubicación de la obra presenta la ventaja de ser a parte de aforamiento más superficial de dicho banco. El proyecto ofrecía dos alternativas: una, en la que se admite suficiente masa de hormigon para asegurar la estabilidad por gravedad, y otra, en la que la subpresión aliviaría la carga, pero previendo un desagüe en el que la altura máxima del nivel hidrostático quedara asegurada. Los dragados y el hormigonado se han llevado a cabo con medios auxiliares potentes y modernos, y los rellenos se han efec-
tuado siguiendo procedimientos hidráulicos.

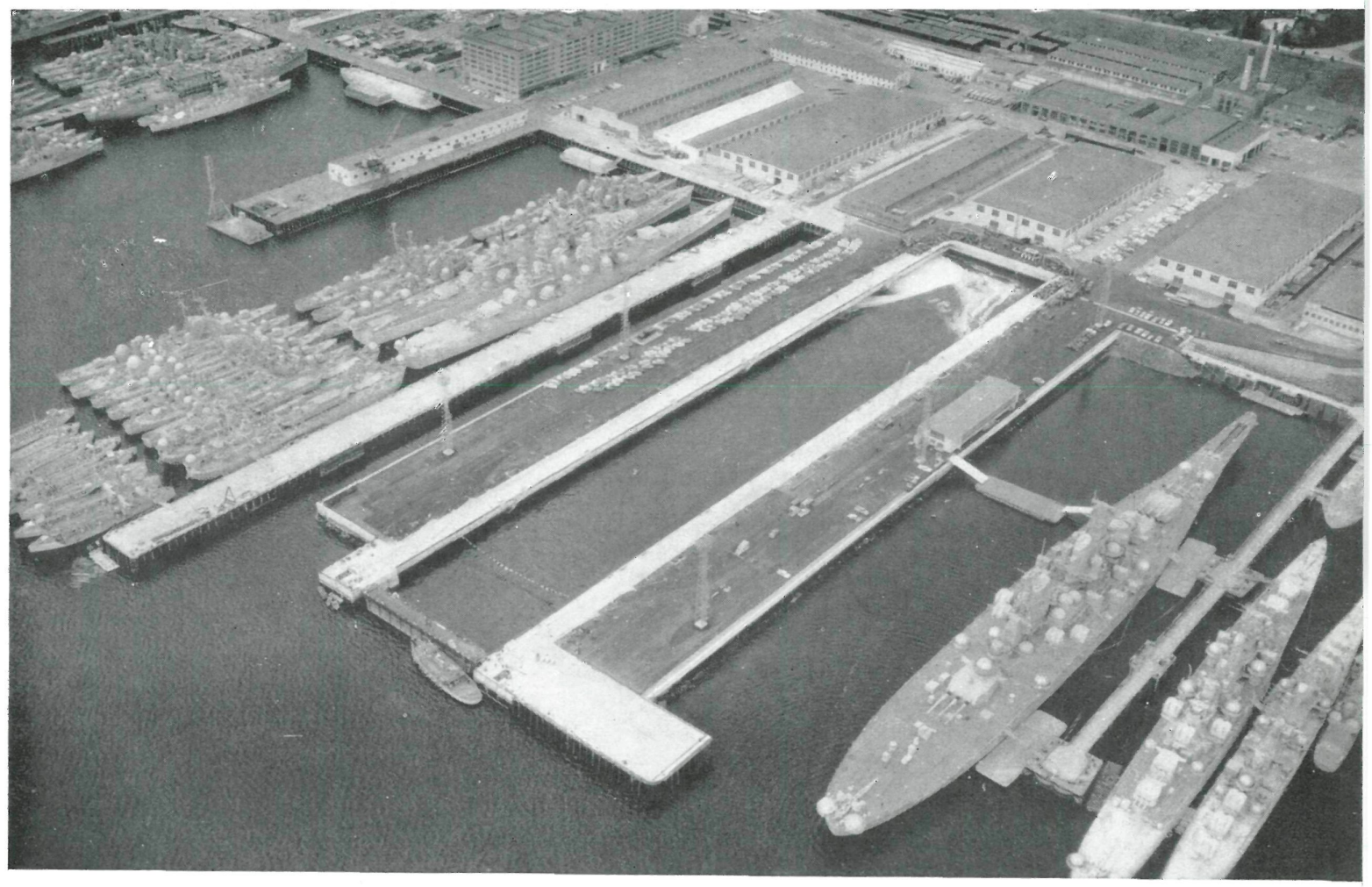

Los portaviones modernos han alcanzado dimensiones inusitadas y, por tanto, los diques actuales resultan pequeños para ellos. Esto ha exigido a la marina americana la construcción de un dique seco en Bremerton, Wáshington (EE. UU.), capaz de admitir a estas unidades navales de gran eslora, manga y calado.

El nuevo dique tiene, en planta, $360 \times 55 \mathrm{~m}$ y una profundidad de $18,50 \mathrm{~m}$. Esta obra constituye el núcleo de las instalaciones actuales dedicadas a la reparación de navíos de la Armada de EE. UU.

\section{el progerefo}

El lugar de ubicación de la obra fue motivo de dilatados y profundos estudios antes de llegar a la conclusión definitiva. Las distintas soluciones presentaban problemas similares en cimientos. El banco admitido como firme para la sustentación está formado por una capa sedimentaria de aluviones glaciares de granulometría discontinua, recubierta por otros materiales fangosos que se han tenido que dragar. Fue determinante para fijar la ubicación la cota de los afloramientos del mencionado banco.

Del estudio de las cargas que podrían admitirse y de las características del suelo se llegó a la conclusión que debían estudiarse las dos siguientes soluciones en el proyecto del dique:

a) El dique podría ser de gravedad, con masa suficiente de hormigón para alcanzar el peso necesario que sirva de contrarresto a los efectos del empuje. 


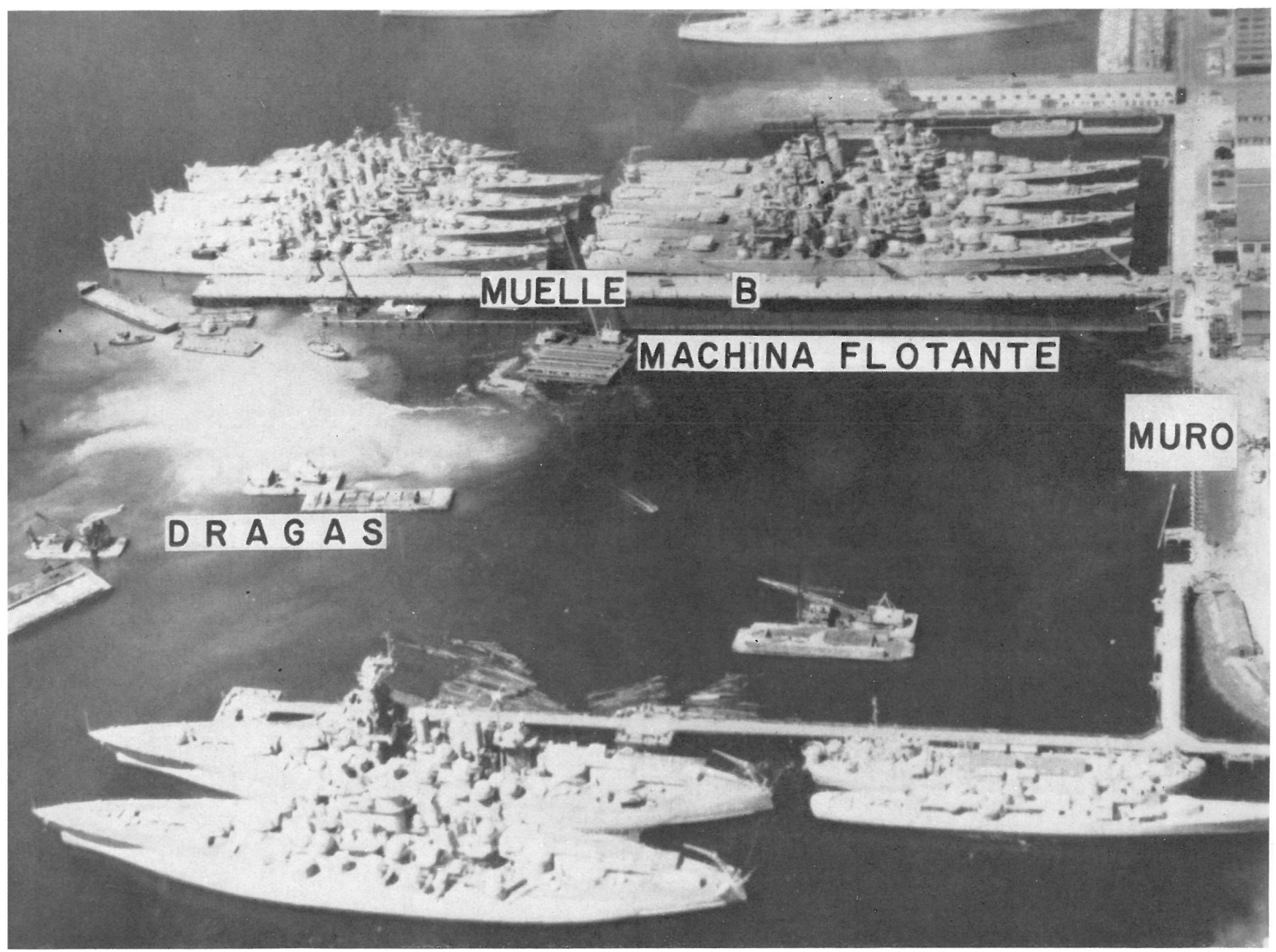

Hinca de tablestacas.

b) Una obra aligerada por flotación que dispondría de suficiente drenaje de fondo y lateral para el desagüe de aguas freáticas, en tal extensión, que el nivel hidrostático no rebosase en ningún caso una altura relativa determinada y cuyo efecto se notase en el fondo y lateralmente.

La primer solución exigía una losa de fondo de unos $13 \mathrm{~m}$ de espesor para tener suficiente peso, pero por distintos motivos fue desechada. Los estudios subsiguientes se encaminaron hacia una solución aligerada. La última concepción requería especial cuidado para asegurar, contundentemente, la perfección del funcionamiento de drenaje, particularmente durante el agotamiento del dique.

La losa de fondo, proyectada siguiendo el método de cimentación elástica, resultó con un espesor de 2,10 m en la parte central y aumenta hasta llegar a 3,60 bajo los muros laterales. El empleo de muros con zarpa extensa en voladizo presentaba ventajas sobre los de contrafuertes o de gravedad y, además, no introducía dificultades en el proyecto. La unión entre los muros laterales y la losa de fondo se calculó con ayuda del método de cimentación elástica. Estos muros tienen 3,60 m de espesor en la base, siguiendo en disminución hacia arriba hasta su coronación, donde el espesor es de $0,75 \mathrm{~m}$ aproximadamente.

El aligeramiento por flotación se consigue por un desaguie continuo del agua de infiltración a través de una capa granular de drenaje debajo del dique y muros laterales, así como a través de un relleno granular alrededor de los muros. Estas capas granulares de drenaje adquieren particular importancia, pues han de asegurar características propias de filtro y una permeabilidad conveniente. Estos factores, acompañados de las características granulométricas de los materiales de relleno, determinaron los datos básicos para fijarlos.

Se utilizó un tablestacado para aminorar las infiltraciones del exterior y servir de apoyo a los materiales de relleno del dique. Debajo de la losa de fondo, y en el seno de la capa de drenaje, se ha colocado un sistema reticulado de tubos perforados que terminan en conductos colectores que atraviesan toda la longitud del dique. También se ha empleado un sistema similar de drenaje en la parte exterior de los muros. A partir de dichos conductos el agua de infiltracion se mueve por gravedad y va a parar a los sumideros de bombeo automático que agotará continuamente el agua de infiltración.

El dique seco se llena por medio de un flujo hidráulico, movido por gravedad a través de conductos embebidos en los muros, que terminan en las aberturas practicadas en el fondo del dique. Esto constituye un sistema mucho más simple que el que se ha utilizado generalmente hasta ahora. 
El tiempo previsto para llenar el dique es de 90 minutos, incluyendo los 20 minutos del período de control al iniciar la operación. La cantidad necesaria para llenar el dique es de 333.000 metros cúbicos.

Los dos principios básicos en que se apoya el proyecto son los siguientes:

1) El tiempo de agotamiento será el mínimo posible para mantenerlo dentro de los límites económicos, consiguiendo con esto reducir el tiempo de maniobra del equipo encargado del agotamiento, así como los costos de energía eléctrica.

2) El equipo de agotamiento está suficientemente perfeccionado para reducir los costos de conservación, eliminando, de tal suerte, el número de piezas de recambio, de forma particular y exclusiva para este tipo de equipo mecánico.

Las cuatro bombas principales se han instalado en una cámara situada a una cota inferior al fondo del dique. Esta cámara de bombeo se ha construido en uno de los muros. Cada una de estas bombas se une directamente al dique, en lugar de hacerlo en un sumidero común a varias unidades, como sucede en otros casos. Con ello se evitará toda interferencia en el régimen hidráulico. La descarga de las cuatro bombas se realiza a través de un conducto común que desemboca en la bahía. La descarga del dique se verifica en 3 horas y 45 minutos.

El equipo mecánico necesario para atender a las necesidades de las reparaciones ha exigido se instale una serie de maquinaria auxiliar variada, entre las que figuran: el bombeo de agua salada para incendios y lavados, agua potable, aire comprimido, oxígeno, vapor, combustibles líquidos, alcantarillado, electricidad, generación o transmisión de energía, sistema de alarma, servicios telefónicos y de reflectores.

Para las canalizaciones de los servicios se han dejado unos conductos en la parte superior e interior de los muros. Estas galerías, de $2,5 \times 2,5 \mathrm{~m}$ de sección, permitirán un fácil acceso para las reparaciones y posibles modificaciones.

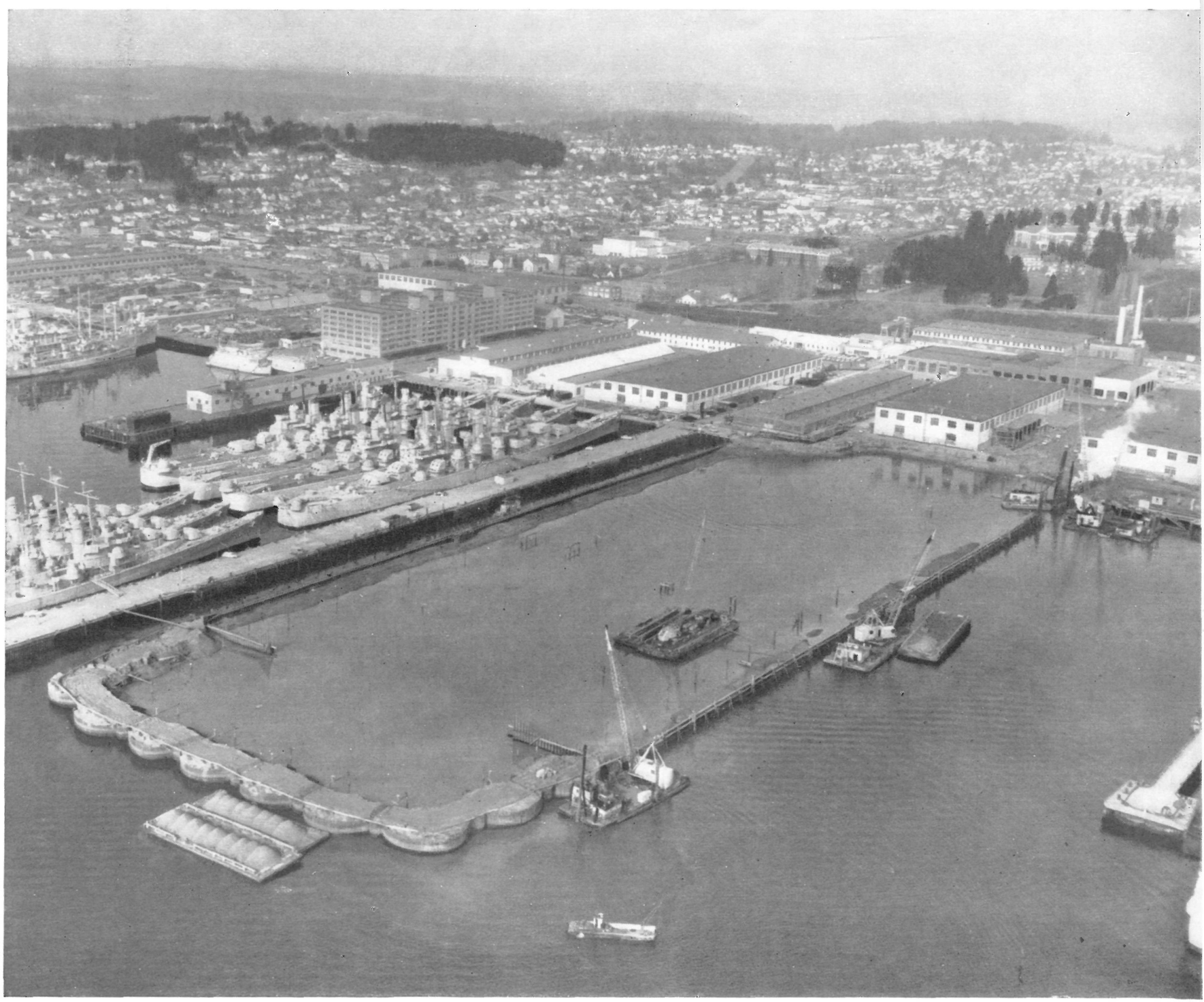

Terminación de la ataguía. 


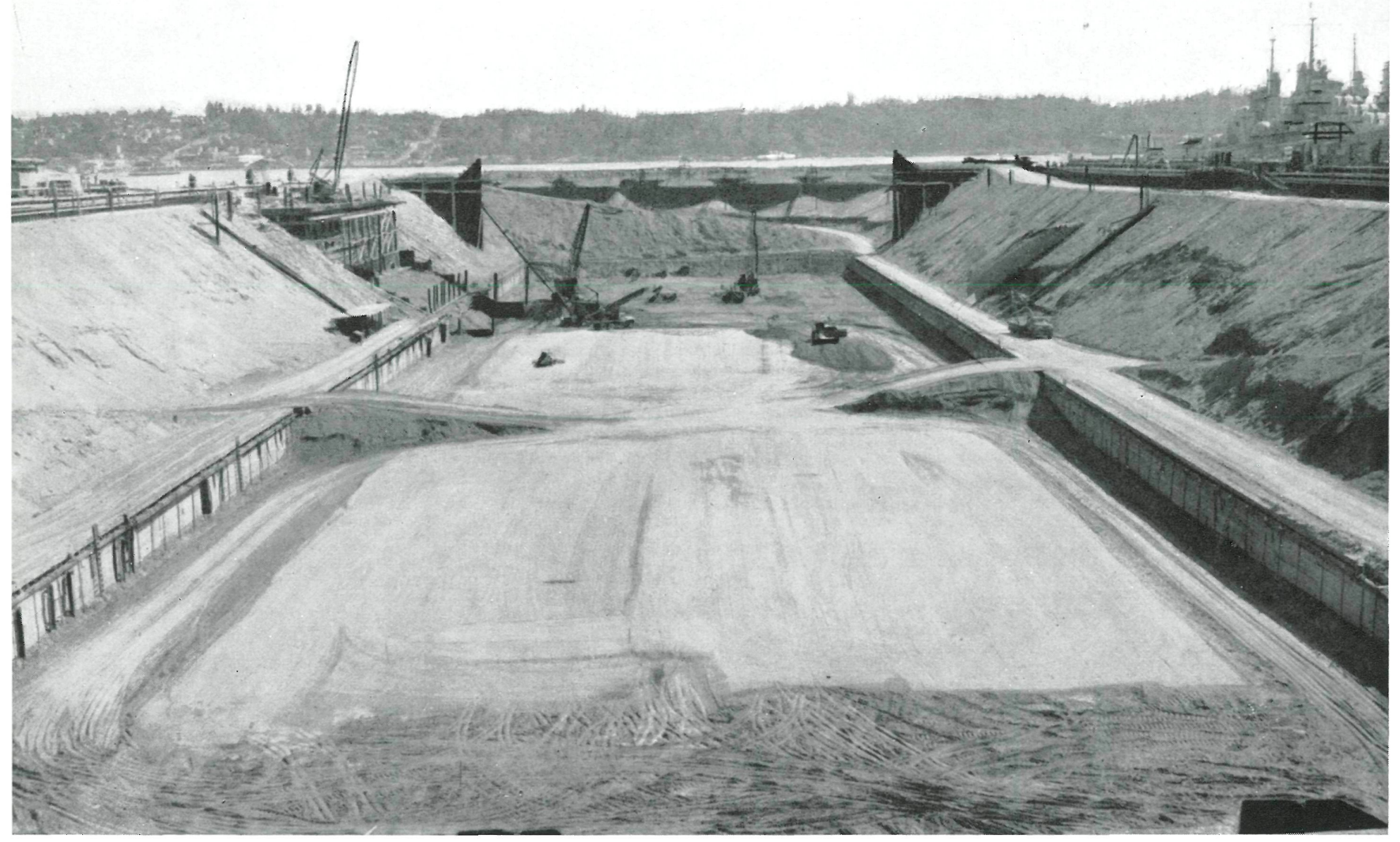

Caminos provisionales para el vaciado.

A lo largo de dos galerías continuas laterales al dique se han instalado, espaciadas a $45 \mathrm{~m}$, siete estaciones o subcentrales de servicios. La unión de los servicios eléctricos y mecánicos con el barco en reparación se verificará entre estas subcentrales y la unidad en carena.

Las grúas, de $50 \mathrm{t}$ de potencia, se mueven sobre vías de $6 \mathrm{~m}$ de trocha, que descansan sobre traviesas de madera. Este tipo de soporte o apoyo se basa en el hecho de que siempre debe esperarse un asiento de los materiales de relleno, y de la adaptabilidad a un reajuste de acomodación después del asiento. Esta solución se ha traducido en una gran economía sobre los procedimientos que soportan las vías por medio de pilotes y vigas entre ellos.

Este sistema de soportar vías para grúas de gran capacidad es original en lo que respecta a:

1) Los pernos de anclaje no penetran en la masa del hormigón estructural.

2) Las ranuras para raíles en el hormigón principal tienen mayor anchura en el fondo que en la parte superior, con lo que se consigue mayor resistencia en la entalladura y presentan una acción de arco invertido para resistir a la subpresión.

3) Exigir una mezcla tal al hormigón, con productos aditivos, que se logre una retracción inconsecuente.

Uno de los cajones, utilizado para el cierre, tiene $58 \mathrm{~m}$ de longitud, 18,60 de altura y $6 \mathrm{~m}$ de anchura. Los elementos estructurales del cajón son vigas de alma horizontal en forma de $\mathrm{K}$ y cerchas verticales en forma de $\mathrm{K}$ también. La pieza plana o alma es una parte importante de las jácenas y cerchas. La plataforma superior, con calzada de 3 m, se ha proyectado para una carga $\mathrm{H}-20$.

En el proyecto se han introducido modificaciones respecto a la disposición estructural de otras obras vecinas y mejoras en los servicios de suministros de combustibles, pues se dispone de una instalación capaz para $141 \mathrm{~m}^{3}$ por minuto y varios tipos de cabrestantes. Las ataguías, entramados auxiliares y retención de taludes fueron objeto de estudio y detalle en el proyecto. El proyectista previó también una secuencia de fases que debían seguirse durante el período de ejecución. Esta secuencia constituyó una cláusula en el contrato de adjudicación, con objeto de reducir las posibilidades de ruina que han ocurrido en otras obras marítimas de este tipo.

\section{CEm}

Los dragados necesarios se realizaron por el procedimiento de succión y con dragas montadas sobre pontones, provistas de cucharas bivalvas. Los materiales de relleno se clasificaron con tamaño máximo de 7,5 $\mathrm{cm}$ y con menos del 10 por 100, pasandn por el tamiz de 100 mallas. Los rellenos se colocaron partiendo de barcazas provistas de costeros móviles que se abrían para descargar el material en los lugares indicados. 
En cimientos se han empleado unas 3.000 t de pilotes de sección en forma de $Z$, hincados en línea a lo largo del dique, para retener las bermas. Las tablestacas tenían longitud variable de 18 a $33 \mathrm{~m}$, y se hincaron unos $3 \mathrm{~m}$ en el interior del terreno natural no perturbado. Las células cilíndricas de la ataguía absorbie ron $2.400 \mathrm{t}$ de tablestacas. Las once células de la entrada se rellenaron con los mismos materiales utilizados para el relleno. Los materiales de su interior se aprovecharán, al desmontarlos, para el relleno de la escollera exterior.

El contrato exigía que las estructuras de la estación de bombeo y toma de agua se realizasen en seco y en el interior del tablestacado de la ataguía. El sistema de agotamientos se debía someter a la aprobación de la autoridad encargada de la obra, pero sin que esto eximiera al contratista de la responsabilidad consiguiente.

Al descender el nivel interior del agua se exigió que el agotamiento se efectuase de tal manera que el nivel freático tuviese una cota no superior a $4 \mathrm{~m}$ sobre dicho nivel. No se permitieron en ningún momento las fugas en las canalizaciones del interior de los taludes dejados durante la construcción, ya que esto podría poner en peligro la estabilidad de dichos taludes. Al alcanzar el agotamiento, el nivel freático en las proximidades del fondo seco interior a la ataguía debía permanecer por lo menos a una cota de 0,60 $\mathrm{m}$ por debajo del nivel de la excavación.

A lo largo del perímetro del dique se colocaron 66 bombas entubadas profundas y de potencia variable de 3 a $20 \mathrm{HP}$. Estas bombas tenían por objeto agotar el agua retenida en las bermas y las aguas de infiltración, estimadas en 75 a $115 \mathrm{~m}^{3}$ por minuto, así como lograr un descenso de $18 \mathrm{~m}$ en el nivel freático. Con objeto de controlar el nivel del agua se colocaron 18 tubos piezométricos con carácter auxiliar durante el período constructivo.

Al terminar de retocar los taludes se instalaron 400 pocillos sumideros, cuyo nivel de caldera fue aproximadamente el correspondiente al fondo del dique. Los colectores y sumideros se pusieron en el exterior de la estructura del dique $\mathrm{y}$ al pie de los taludes retocados. No fue necesaria la colocación de sumideros intermedios formando anillo, ya que se dispusieron bombas entubadas profundas para el agotamiento del agua retenida en los pozos de la berma. Por todo ello, el retoque final de taludes se facilitó considerablemente y se aceleró mucho la construcción. Tanto las cabezas de los sumideros como de las bombas entubadas se hallaban en un banco compacto de arena gris, con grava, que resultó ser el más favorable para la infiltración del agua.

La infiltración definitiva era inferior a la prevista. El volumen de agua inicial disminuyó considerablemente después de la instalación de los sumideros profundos. Las pendientes de los taludes ajustados varió de $1: 23 / 4$ a $1: 13 / 4$

Los escombros de la excavación se amontonaron en el exterior del dique por medio de cintas transportadoras. Estos materiales se utilizaron para rellenar el espacio entre el muro del dique, al exterior, y las bermas.

Al terminar la escavación se puso una capa de 0,30 m de espesor, de materiales secos, que después se com pactaron con cilindros de ruedas de goma, de $50 \mathrm{t}$, movidos por tractores. En algunas zonas se pudo comprobar la existencia de una ondulación en los materiales consolidados, que parecían ceder ante el paso del cilindro y después emerger nuevamente. En una profundidad de $0,30 \mathrm{~m}$, la compactación era buena, pero el conjunto del fondo parecía como si flotase.

Una serie de nuevos sondeos pusieron de manifiesto que los lodos del río habían desaparecido de los materiales de relleno, a pesar de lo cual éstos tenían un grado de finura superior al permitido por las normas. La consolidación del terreno y la capacidad de sustentación, juzgada por el número de golpes sobre el dispositivo de prueba, es decir, de 2 a 3 golpes por cada $0,15 \mathrm{~m}$ de penetración en algunos casos, era demasiado baja para que se pudiese aceptar.

Para reforzar el suelo de apoyo en cimientos se consideraron varios métodos, tales como asiento forzado por explosivos o la hinca de pilotes. El primero se desechó por poder perjudicar a la ataguía, y el segundo por ser demasiado caro. El equipo técnico propuso un proceso de vibroflotación como solución práctica del problema.

En todos los lugares donde la capa de rellenos era de más de $2,50 \mathrm{~m}$ de espesor se empleó el procedimiento de vibro-flotación (vibración y saturación simultánea de los materiales); para ello se colocaron 848 vibradores espaciados a unos $3 \mathrm{~m}$ y a una profundidad de $3,40 \mathrm{~m}$. Por término medio se añadieron unos $170 \mathrm{l}$ de nuevos materiales por cada $0,30 \mathrm{~m}$ de taladro; en total, unos $2.500 \mathrm{~m}^{3}$ de arena y grava, pero el nivel de la superficie descendió.

De las observaciones de los niveles, antes y después de la compactación, se desprende que el asiento ha sido del orden de $1 / 10$.

La adición de 701 de materiales por cada $\mathrm{m}^{3}$ del total de materiales tratados, más $1 / 10 \mathrm{~m}$ de asiento, significaría una densificación equivalente a añadir unos $125 \mathrm{~kg}$ de materiales a cada $\mathrm{m}^{3}$ de material tratado.

Si admitimos un peso específico de $1.842 \mathrm{~kg} / \mathrm{m}^{3}$ para los materiales secos antes del tratamiento, un mínimo de $1.600 \mathrm{~kg} / \mathrm{m}^{3}$ y un máximo de $2.082 \mathrm{~kg} / \mathrm{m}^{3}$ y estimamos la densidad relativa de 50 por 100 antes del tratamiento, entonces la adición de $125 \mathrm{~kg} / \mathrm{m}^{3}$ supondría un aumento de densidad relativa de 50 al 75 por 100 . Para lograr este mismo efecto a través de la consolidación, el dique seco, si se hallase soportado por estos materiales, experimentaría un asiento medio de unos 22 centímetros. 
Partiendo de los resultados obtenidos y las mediciones realizadas se pudo comprobar que la compactación por vibro-flotación influenció la zona media entre los taladros para ella preparados, logrando la consolidación máxima donde más se necesitaba, es decir, en la parte más profunda de los rellenos más finos. La densidad lograda en los puntos más desfavorables fue del 60 al 65 por 100, y en el propio taladro se estimó de 85 al 90 por 100.

Terminada la consolidación se colocó una capa de 0,85 $\mathrm{m}$ de espesor, de grava elegida, que serviría de drenaje y que se compactó al 100 por 100 de densidad con humedad óptima. En esta capa se colocó un reticulado de tubos de gres y por encima se protegió con una lámina de politileno. El sistema de drenaje se protegió con una capa de hormigón armado, de $10 \mathrm{~cm}$ de espesor, que también serviría de plataforma de trabajo. La solera del dique está constituida por bloques de hormigón de 2 a $3,40 \mathrm{~m}$ de espesor.

Se utilizaron encofrados metálicos, y se transportaron por medio de mesillas montadas sobre raíles. El hormigón se preparó en una Central situada a unos $400 \mathrm{~m}$ del dique.

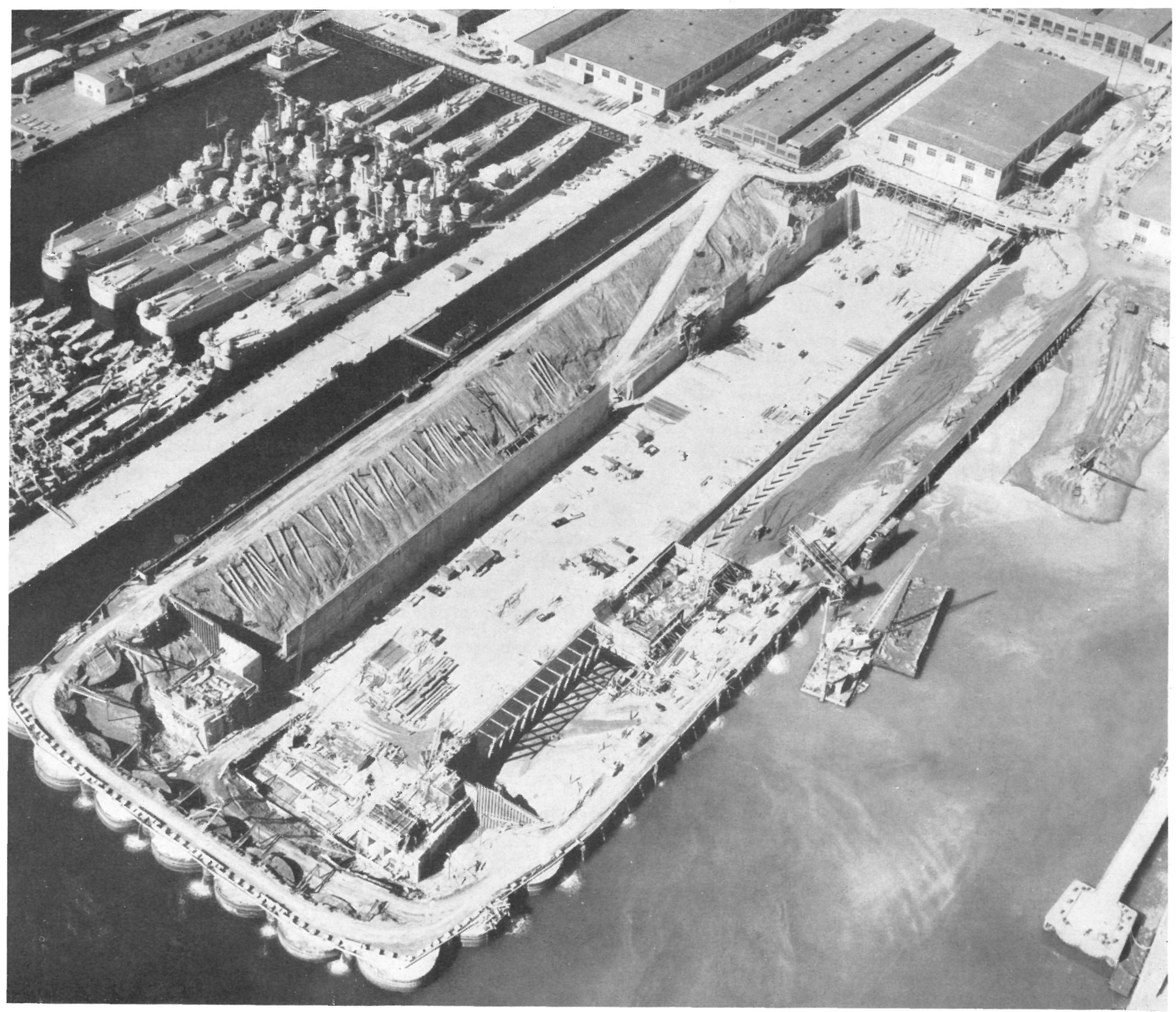

Construcción de muros laterales. 


\section{Un boussin de radoulb}

Information fournie
par Northwest Division-Bureau of Yards and Docks.

Les grands porte-avions modernes ont des dimensions telles que leur réparation devient difficile dans les bassins normaux. dimensions sont de $360 \mathrm{~m}$ de longueur, $55 \mathrm{~m}$ de largeur et $18,50 \mathrm{~m}$ de profondeur, situé près des chantiers navals dont dispose la Marine à Bremerton.

Au cours de la première phase de ces travaux a été construit une digue de travail, d'environ $48 \mathrm{~m}$ de largeur, de chaque côté du futur bassin, et un quai d'accostage pendant la seconde.

Les installations modernes dont est doté ce nouveau bassin couvrent tous les besoins qu'exige ce type de réparations et feront de cet ouvrage un ensemble opérationnel économique et efficient.

Plusieurs solutions et emplacements furent étudiés, car le fond de la baie présentant diverses couches de fanges et de sables de différente granulométrie laissait prévoir de possibles difficultés pour les fondations. La couche supérieure, admise omme base acceptable pour la sustentation del'ouvrage, est composée d'un banc sedimentaire de détritus glaciaires. L'en-

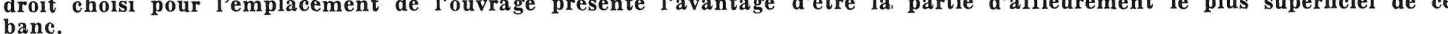

Le projet offrait deux alternatives: l'une pour laquelle était admise une masse suffisante pour assurer la stabilité par maximum du niveau hydrostatique serait assurée.

Le dragage et le bétonnage ont été exécutés à l'aide de puissants moyens auxiliaires modernes et les remplissages ont été effectués suivant des procédés hydrauliques.

\section{Dreg Dock for the U. S. Meny}

This information

has been supplied by the Northwest Division, Bureau of Yards and Docks.

The great modern aircraft carriers are so large that it is difficult to repair them in the existing dry docks. This has made it necessary to build a new dock, suitable for these huge ships. The new dock is $360 \mathrm{~ms} 10 \mathrm{lng}$, $55 \mathrm{~ms}$ wide and $18.5 \mathrm{~ms}$ deep and is situated at the Navy Dockyards in Bremerton.

In the initial phase of this project two working docks, $48 \mathrm{~ms}$ wide, were constructed on each side of the intended dock. A second phase of the work includes the construction of the mooring wharf.

The modern installations of the new dock will provide all that is necessary for the repair of the carriers, so that the dock and its ancillaries will constitute a vast economic and functional unit.

Various designs and locations were initially studied, as the bottom of the bay has layers of mud and sand of varying texture, which indicated possible difficulties in constructing the foundations. The highest stratum which was regarded as acceptable for supporting the dock consists of a layer of sedimentary glaciar detritus. The dock was finally located at the highest part of this soil formation.

The project implied two alternatives. In one of them a sufficient concrete mass would be used to ensure the stability of the structure due to gravity, and in the other, the water pressure would take part of the load of the dock, although the dock would be able to fill up and empty efficiently.

The dredging and concreting have been carried out with conventional, though powerful and up to date, equipment, and fill material has been placed by hydraulic means.

\section{Trockemedock}

Nachricht verschafft

durch Northwest Division - Bureau of Yards and Docks.

Die grossen modernen Flugzeugträger haben derartige Ausmasse, dass ihre Reparatur an gewöhnlichen Dämmen erschwert wird. Dies hat den Bau eines neuen, für jene Schiffsart fähigen Dammes erfordert, der $360 \mathrm{~m}$ lang, $55 \mathrm{~m}$ breit und 18,50 m tief ist und nahe den Schiffswerften liegt, über welche die Kriegsflotte in Bremerton verfügt.

Bei der ersten Entwicklungsstufe dieser Arbeiten wurde ein etwa $48 \mathrm{~m}$ breiter Hafendamm zu beiden Seiten des künftigen Docks für die Arbeiten erbaut. In einer zweiten Entwicklungsstufe wird ein Anlegedamm gebaut werden.

Die modernen Anlagen, mit denen das neue Dock rechnen wird, decken alle Hauptbedürfnisse, welche diese Art von Reparaturen erfordern, was das Ganze zu einer wirtschaftlich und wirkungsvoll tätigen Gesamthelt machen wird.

Es wurden verschiedene Lösungen und Plätze zur Aufstellung studiert, da der Grund der Bucht mehrere Schlamm- und Sandschichten von unterschiedlicher Korngrösse aufweist, welche mögliche Schwierigkeiten für die Gründung voraussehen Ablagerungen gebildet. Der ausgewählte Platz für die Lage des Werkes hat den Vorteil, dass er sich am höchstgelegensten Teile des Aufschlusses der genannten Bank befindet.

Das Projekt bot zwei Doppelwahlen: bei einer lässt man eine genügende Betonmasse zu, um die Stabilität durch die Schwere zu sichern, und bei einer anderen würde der Auftrieb die Belastung erleichtern; jedoch würde man einen Wasserablauf vorsehen, womit die maximale Höhe des hydrostatischen Niveaus gesichert bliebe.

Die Nassbaggerungen und das Betonieren werden mit leistungsfähigen und modernen Hilfsmitteln und die Füllungen nach hydraulischem Verfahren ausgeführt. 